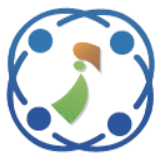

\title{
Path Load Balancing Adaptive Gateway Discovery in MANET-Internet Integration Using PSO
}

\author{
Jay Prakash ${ }^{1 *}$, Rakesh Kumar ${ }^{2}$, Jai Prakash Saini ${ }^{3}$ \\ 1,2 Department of Computer Science \& Engineering, \\ Madan Mohan Malaviya University of Technology Gorakhpur, India \\ ${ }^{3}$ Department of Electronics and Communication and Engineering, \\ Bundelkhand Institute of Engineering and Technology, Jhansi, India \\ * Corresponding author's Email: jpr_1998@yahoo.co.in
}

\begin{abstract}
Mobile Ad-hoc Networks (MANETs) find numerous applications. Its utility can be extended by integrating it with Internet. Some real-life applications where such integrated networks can be used are disaster situation viz., earthquake, battle fields etc. MANETs are dynamic topology, infrastructure less and standalone networks of wireless mobile nodes. To overcome this limitation, MANET needs to connect to the Internet. In such networks, mobile nodes do not have wired connection, so they do not follow any particular route to transfer their packets. Thus, every time when topology changes, routing paths changes too. However, every node selects the shortest path to route their packet. In that case, this path will be congested due to overload. We propose a load balancing adaptive Internet gateway discovery approach which focuses on solving the problem of overload and congestion in mobile ad-hoc domain. Particle Swarm Optimization is used for selecting an optimal path among all paths available. Our work is to optimize this load balancing problem. We are using Particle Swarm Optimization (PSO) concept to overcome this problem. In our approach, we use soft computing technique viz., working procedure of PSO algorithm which searches the optimal paths using some mathematical function. Then, we combine shortest path algorithm with the gateway discovery algorithm. Thus, the algorithm first selects the shortest path stored in the memory and check whether it is free for routing packets or not, if the route is free or lightly loaded, then the source node will transfer packets otherwise if the node is overloaded, then PSO searches for the next optimal neighbour or node to route the packet. The new path is selected to route packets and stored in the place of current available path. Proposed algorithm has been implemented in MATLAB. Our approach outperforms the existing approaches.
\end{abstract}

Keywords: Mobile ad hoc network, Path load, Swarm optimization, Shortest path, Performance.

\section{Introduction}

Ad-hoc networks [1] can be defined as the network infrastructure where the mobile nodes are connected to each other wirelessly. The mobile nodes such as laptops, mobile phones, digital devices and all the personal assistance devices. These mobile devices communicate through internet gateways for transferring data packets to each other via wireless connection. Whenever a mobile node wants to communicate or transfer data to another mobile node it searches for gateways. When the gateway responses for their request they transfer their data via routers. Routers are also mobile device which only perform the data transformation coming from one node to another node. A router does not only help to transfer the data packet from source node to destination node. They always select the shortest path to route the packet so that time delay will be less and bandwidth will be more utilized. These mobile nodes move their position frequently thus the topology change very often in mobile Adhoc networks. So, the path which is selected once to route the packet can change even though the secondtime source and destination nodes are same or different. Topology change is one of the main 


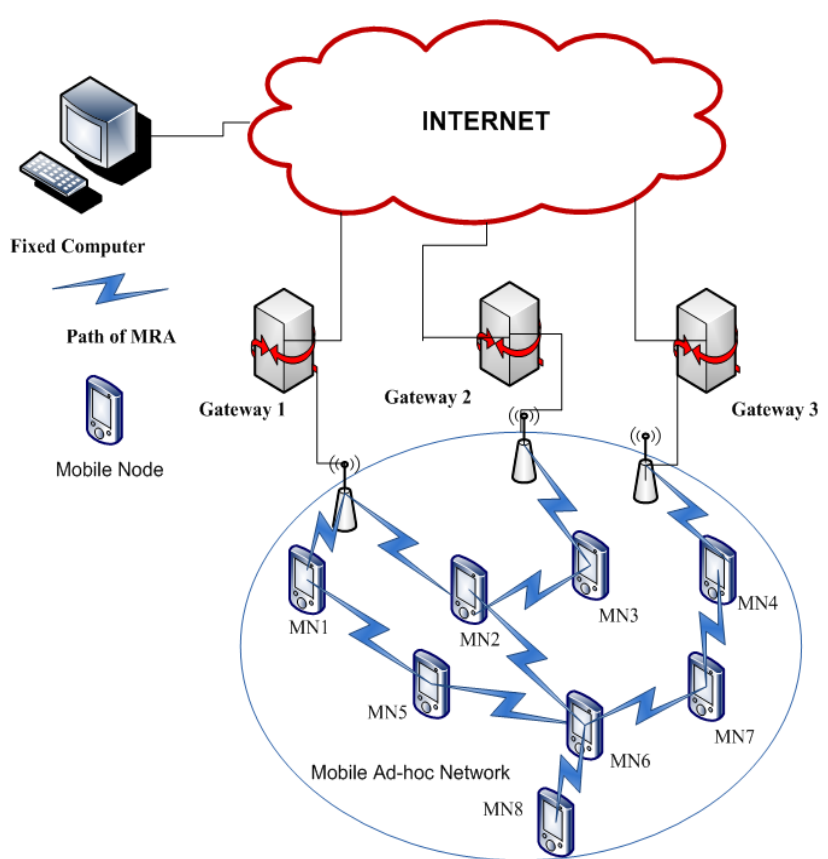

Figure.1 Architecture of Integrated Internet-MANET

characteristics of the Ad-hoc network. Fig. 1 shows the architecture of mobile Ad-hoc network. For transferring data packets, first it searches for all the available paths, then select the shortest path from source to destination node. As every node wants to route their data from the shortest path only, thus the path becomes congested one.

Our main concern is to reduce the load on the path and balance the load on the Ad-hoc network. We are using Particle Swarm Optimization [2] to minimize the path load. Particle Swarm optimization (PSO) is a pretending optimization technique which is based on population based problem. Here, we are using shortest path algorithm with the combination of PSO.

Organisation of research paper is as follows. In current section an overview of working culture is presented. In section 2 literature review of existing works is described. Basic concept of particle swarm optimization is presented in section 3. Section 4 includes proposed work that contains algorithm for path selection using PSO and also algorithm for gateway discovery. In section 5 simulations and result analysis is presented and finally in section 6 conclusion and future work are described.

\section{Related work}

There are various issues of load balancing in an ad-hoc networks. In the following section, we analyze many survey and research papers where authors discussed and gave various solutions to deal with load balancing problem.
The technique given by Zhou et al. [3] consists of a dynamic GW (DGW) and a foreign agent with a distance of one hop in them and the DSDV protocol is used for data transmission. DGW solves the problem of load balancing. The registration is done in two ways proactive in this registration is between DGW and FA and reactive in this in between DGW and mobile nodes. The registration contains current queue length and no. of nodes registered. The selection of DGW and FA is emphasized by the load balancing. This approach consists of load balancing DGW selection. The DGW though improves the process but also complicates it.

The technique proposed by Trivino-Cabrera et al. [4], consists of an access router AR and the nodes in its range are default gateway(DLW) and the nodes away from it are the candidate gateway nodes(CGW) along with these the reactive protocol is AODV. The DGW broadcast MRA message for advertisement and receives the MRS message from other nodes who wants to connect. Whenever the DLW becomes congested mobile nodes becomes the CGW and is used to remove the congestion problem. The drawback of this approach is single point failure.

The technique proposed by the Hsu et al. [5], consists of two tier architectures. In first tier is MANET provided with services from DCHP server which acts as a gateway, this forms the lower level of DSDV. The mobile nodes can also get the valid IP address by DCHP server. The DCHP server might not be in the MANET. There are two categories based on DCHP server presence and absence. The DCHP server may be present or absent in MANET. The first is sub-MANET with DCHP server and other is disconnected component without DCHP. The second tier have heterogeneous cellular infrastructure. The GW is connected with one base station and DCHP acts as GW and registration is by DCHP protocol. The GW selection is by minimum load index routing (MLIR). This protocol decides the gateway and mobile node based on load and traffic and proves that range may expand or shrink on traffic basis. The limitation is that the handoff mechanism is not well supported.

The technique proposed by Shin et al. [6] classifies the traffic in external and internal traffic. If the both source and destination are in ad-hoc network then it is internal traffic else external traffic. This external traffic has two types- incoming and outing so for it two GW are maintained. The GW having least distance is default GW but congestion may occur so registration is done to avoid it with load balancing. The ad-hoc network uses reactive routing protocol in which the node sends request and GW sends the proxy route reply message (pRREP). 
The load balancing mechanism is combined with delay of sending the p-RREP. The mobile node will receive p-RREP of the GW. For receiving the message reactive method is used to send solicit message to $\mathrm{GW}$ and registration to it is done. For measuring the load of GW, the average_packet_queue_size (APQS) is used.

The technique proposed by Le-Trang et al. [7] divides network into MANET domains each having a GW which manages the network topology. GW registration is necessarily done and GW broadcast advertisement like in proactive scheme and the selection of GW is on the basis of load on gateway. Limitation is that, during the performance evaluation based on average delay transmission factor, AODV routing protocol should be used for decrementing delay ratio.

In paper [8], authors have presented a congestion adaptive multipath routing protocol for enhancing the throughput and eliminates congestion in MANETs. Here, the average load of an existing link increases beyond a defined threshold. The available bandwidth and residual battery energy decreases below a defined threshold, traffic is distributed over fail-safe multiple routes to reduce the traffic load on a congested link. Demerit of this approach is that, author estimate each threshold value individually for link presence in network, on behalf of selecting a single threshold for all link in network.

Pham et al. [9] suggested a wireless multi hop network. In this, the Internet Gateway (IGW) mechanism is adopted to obtain Internet connectivity, linking the wireless network with the global Internet. The efficiency of the protocol can be enhanced if it balances the traffic among available IGWs by which the network performance can be optimized. In this proposed approach three type of overhead faced such as tunneling overhead, additional cost and last is selection of gateway, which are not be consider by author. This is major drawback of this approach.

In paper [10], authors have proposed a modified version of AODV where a new gateway load balancing strategy is based on a load balanced version of AODV routing protocol. The network provides Internet connectivity to mobile nodes which form a mobile ad-hoc network by using a set of Internet Gateways (IGW). With the aid of IGW, mobile nodes can connect to the outside world and wired node like correspondent Node $(\mathrm{CN})$. Thus, two protocols are run by IGW, IP for wired network and modified-AODV for the wireless ad-hoc network. Throughput and load balancing factor are increased but due to considering a very small size buffer the packet drop ratio is dramatically increased, which is the drawback of this modified work.

In paper [11], authors have proposed a load balancing scheme in ad-hoc networks. The proposed mechanism is designed which is compatible with adhoc on-demand distance vector routing (AODV) protocol. In this mechanism, each nodes within a MANET checks its queue occupancy continuously in order to determine whether or not it should respond against received route requests (RREQ). This decision is totally relying on a threshold value and this value for threshold is adjusted adaptively as per of network load conditions. The limitation of this scheme is that, there are variations among the threshold value during the forward registration and backward reply process.

The technique given by Gunes et al. [12] uses Ant-Colony based Routing Algorithm that uses route discovery by flooding as in case of AODV. The duplicate packets are identified using sequence number. When we found a route to the destination, and we found backward ant like RREP in AODV. The ant follows backward path with shortest trip time by detecting the forward ant. The pheromone amount deposited by ant is a function of route length. The route maintenance is responsible for managing the routing information.

$\mathrm{Hu}$ et al. [13] presents a review paper which describes Particle Swarm Optimization Algorithm in detail. It discusses the basic concept of PSO and all the parameters used in it. Author details the algorithm used in PSO and explain how the algorithm searches the optimize value in the dimensional space.

Zaman et al. [14] presents an integration of adhoc network with the Internet through Internet Gateway (IG). The IG offers Internet services to the mobile nodes within ad-hoc domain. Prior to connecting with Internet, there are two steps involved. Firstly IG discovery and then registration. Numerous strategies are defined for gateway registration such as proactive, reactive and hybrid. Now when mobile nodes communicate with the gateway then due to actual routing of the data path towards gateway result in overloaded. To overcome this problem, the author's proposed PLB-MSC (Path Load Balancing Scheme for Maximal Source Coverage). In this paper, PLB-MSC algorithm is considered that helps in adjusting the load of the network by deviating network traffic towards less congested route and at the same time it dynamically alter the range of the gateway advertisement message, so as try to eliminate the unnecessary control message in the ad hoc network. This combined system present maximal source coverage 
strategy in which path load balancing and the adaptive gateway discovery both issues are discussed simultaneously. Maximal source coverage strategy truly adaptive path load balancing mechanism for that there is need to enhance the range of the Gateway advertisement message dynamically.

Khan et al. [15] presents a method for selection of an efficient gateway and routes to other mobile nodes two factors were consider in this approach first is length of the routing queue and other is minimum hop count. This is based on the proactive gateway discovery approach. It is a novel approach implemented by calculating the path load and hence updating the routing table entry whenever there is a route request from one node to other. The newly updated routes can be used without any prior waiting for advertisement. There is reduction in the delay along the path for packet transmission. As here there is selection of less congested path it will help in increasing the throughput. There is always a relative overhead of managing routing queue and pre-advertisement route maintenance.

The above-mentioned schemes have used the different schemes like the candidate gateway, multitier, network division and some others also and various protocols like the DSDV and AODV were used. In these the gateway discovery process was improved by improving the congestion control tactics but overloading is observed in these approaches. So, to remove this and develop a more efficient scheme the proposed scheme has been developed based on the swarm optimization method.

\section{Particle swarm optimization basics}

Particle Swarm Optimization algorithm is developed by Kennedy and Eberhart in 1995 [16]. This new concept was given to solve the optimization problem and was adapted very vastly and researchers focus more about it and interest gradually increase in last few years. This optimization technique is based on the social behaviour of bird flock or fish school. The PSO finds optimal solution of a problem by updating velocity and position of particle in search space iteratively until the termination criteria is not achieved. In Fig.2, we can see that every cluster having population of various particle searches some optimal particle with whom it can communicate. The working procedure of PSO is that a particle roams in the search space and interacts with other particles to find the optimal solution, in addition to this every particle has their own position and

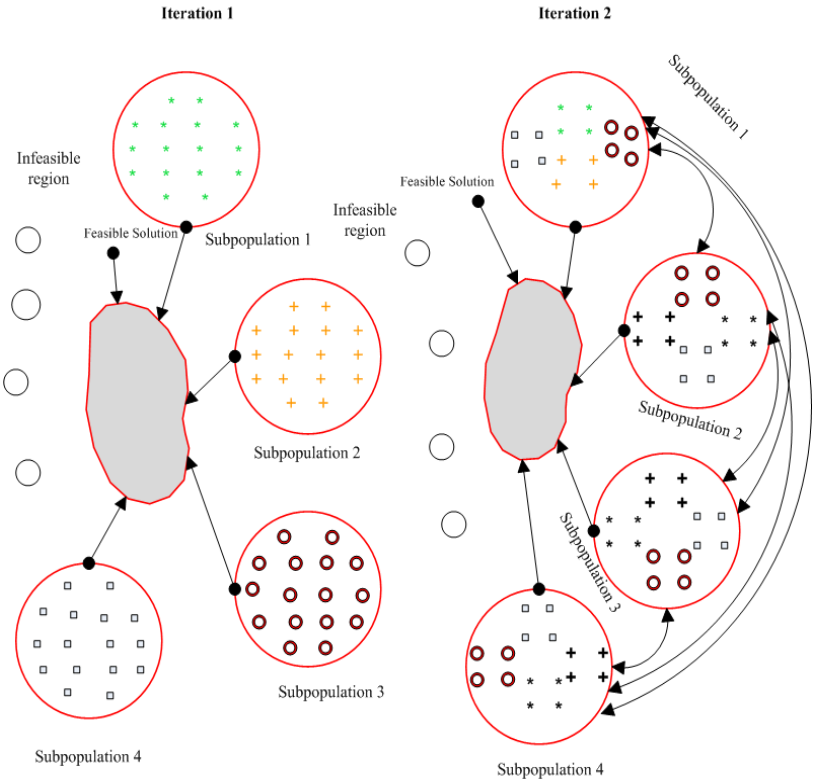

Figure.2 Search model

velocity vector. They also have their global vector to present global optimal solution. Here 'i' represents the particular particle. Each particle stored some memory which contains particle best position and particle global best position.

Particle Swarm Optimization optimizes the function by finding the minimal optimal solution. Here Eq. (1) performs the velocity update and position update. Vid denotes the present velocity of particle with ID number id, c_(1 )and c_2 denote the acceleration constant. Normally we take this constant close to 2 .

$$
\begin{aligned}
V_{i d+1}= & V_{i d}+c_{1} r_{1}\left(P_{B E S T}-X_{i d}\right) \\
& +c_{2} r_{2}\left(G_{B E S T}-X_{i d}\right) \\
X_{i d+1}= & X_{i d}+V_{i d+1}
\end{aligned}
$$

where, $i d=1,2, \ldots n$

$P_{\text {Best }}$ and $G_{\text {Best }}$ are the position best and global best value of the particle respectively. Further, in Eq. (2), $X_{i d}$ represents the position of a particle. These both equations are used in optimization process and velocity and position is adjusted according to this. Algorithm for PSO is discussed below.

\section{Algorithm for Particle Swarm Optimization}

1: Randomly set the position and velocity for each particle.

2: Calculate fitness value of each particle. 
3: Calculate $P i,{ }_{B E S T}$ and $G i{ }_{B E S T}$ for each particle.

4: Iterate following process until termination criteria is achieved

a. Update velocity of each particle by using Eq. (1)

b. Update position of each particle by using Eq. (2)

c. Calculate fitness value of each particle

d. Update $P i{ }_{B E S T}$ and $G i{ }_{B E S T}$ of each particle using following equations-

$$
\begin{gathered}
P_{i, B E S T} \\
= \begin{cases}P_{i, B E S T} & \text { if } f\left(P_{i}\right) \leq P_{i, B E S T} \\
P_{i} & \text { if } f\left(P_{i}\right)>P_{i, B E S T}\end{cases} \\
G_{i, B E S T}=P_{j}
\end{gathered}
$$

Where $P_{j}$ is the position of particle with maximum fitness value and $f(x)$ is the fitness function

Once the iteration will over, the algorithm gives the global best and position best value for every particle in the search space. Now the algorithm compares the value with previous stored global best and position best value in history. If it gets more optimal values then set this current value as present best optimal value for each particle.

To implement the PSO we need an objective function or fitness function which will iterate the value of particle best position. Here, we are assuming a graph $\mathrm{G}$ and the vertices of the graph are represented by $\left(v_{1}, v_{2}, v_{3}, \ldots v_{n}\right)$ and set of node in the graph $G$ are represented by $\left(n_{1}, n_{2}, n_{3}, \ldots . n_{n}\right)$ and edges are represented by $\left(\mathrm{e}_{1}, \mathrm{e}_{2}, \mathrm{e}_{3}, \ldots . . \mathrm{e}_{\mathrm{m}}\right)$ and the load on the edges or the weight on the edges are represented by $\mathrm{W}=\left(\mathrm{w}_{1}, \mathrm{w}_{2}, \mathrm{w}_{3}, \ldots \mathrm{w}_{\mathrm{m}}\right)$. Swarm optimization and the right path selection is given above in algorithm. Thus, to enhance the performance and increase the services in the network the shortest path between the source to destination is calculated by using this objective function in PSO which is given by this Eq. (3).

$$
\begin{aligned}
f\left(P_{i}\right) & =\left(\sum_{j=1}^{N_{i}-1} C_{y z}\right)^{-1} \\
y & =P P^{i}(j) \text { and } z=P P^{i}(j+1)
\end{aligned}
$$

In the above equation $\mathrm{PP}^{\mathrm{i}}$ is the sequential set of nodes in path of particle $i^{\text {th }}$ particle and $\mathrm{N}_{\mathrm{i}}$ is the number of nodes in path of particle i. $\mathrm{C}_{\mathrm{yz}}$ represents the load in path connecting node $\mathrm{y}$ and $\mathrm{z}$.

Now we are defining the procedure, how we are implementing the particle PSO and the right path selection algorithm. Particle Swarm Optimization is used in MANET for selecting appropriate path among all paths available. We discuss the algorithm for path selection using PSO below. In this, CurNode is number of current nodes, $\mathrm{M}$ is the number of neighbours of a particular particle, $\mathrm{N}_{\mathrm{i}}$ is the ith neighbour.

\section{Proposed work}

Here we are developing two different approaches in which first one is used for path selection and second one is used in discovering Internet gateways.

\subsection{Algorithm for path selection using PSO}

Particle Swarm Optimization is used in MANET for selecting appropriate path among all paths available. We have discussed the algorithm for path selection using PSO below.

Optimum Minimum Load Finding Algorithm: N: Number of Mobile Nodes, $\mathrm{N}_{\mathrm{i}}$ : $\mathrm{i}^{\text {th }}$ Neighbour of a node

1: Define N Number of mobile Nodes in the network with specific parameters in terms of energy, transmission rate etc.

2: Define the Source and the Destination node over the network

3: Set CurNode as the current node i.e. Source Node

$$
\text { CurNode } \leftarrow \text { ID of current node }
$$

4. Find M Neighbor Nodes of Nodes CurNode and maintains the respective Information.

5. for $i=1$ to $M$ calculate
a. Distance (Neighbor (i))
b. Load (Neighbor (i))
c. Delay (Neighbor (i))

\section{end for}

6. if Load (Neighbor (i)) < Threshold and

Delay $<$ Threshold 1

/* Reliable node identify*/ 
for $\mathrm{i}=1$ to $\mathrm{M}_{\mathrm{i}}$

CollectInformation( $\mathrm{Ni}$, $\operatorname{Neighbor}\left(\mathrm{N}_{\mathrm{i}}\right)$ ) end for end if

7. Implement Forward SWARM to find the alternate path in each Direction of Neighbour (N (i)).

8. Set the Pheromone on Each Hop and Identify the possible Path

9. Implement Backward SWARM to inform neighbour nodes about backup path

10. Trace the pheromone and communicate to new path.

11. Perform normal communication

The description of the Swarm concept is presented here

- At regular interval, any node $s$ (source) is selected to send data to some destination node d.

- Each forward Swarm selects the next hop node using the routing table information. The next node selected depends on some random scheme. If all nodes already visited a uniform selection will be performed.

- If the selected node is some attack or damage node or it is not currently available. The forward Swarm waits to turn in the low priority node from the queue.

- It will identify any of the next non-visited nodes and pay some delay on it.

- If some cycle detected, the Swarm is forced to turn on the visited node.

- When the Swarm reaches the destination node a backward Swarm is generated to transfer all its memory.

- Backward Swarm uses same path generated by forward SWARM.

Every time to send the packet, a root is selected on the basis of path selection formula and the criteria of selecting the best path is on the basis of which path has lowest load and minimum energy. So that we always select the less loaded path. That criteria will ensure that we always select the path with minimum load. If we get any problem in communicating with next node or selecting the next node for example if all routes are busy in routing or some links are broken then we apply the Particle Swarm Algorithm. The purpose of PSO is to select the same previous path until no other path is available to route the packet. Thus, we achieve load balancing in the ad-hoc network by applying path selection whereas Swarm Optimization algorithm gives the required reliability. Thus, above algorithm will find out the optimal group of node by which we can route data without load creating on path and this path will be more efficient and reliable.

\subsection{Algorithm for gateway discovery using PSO}

In our gateway discovery approach, we use the discovery process based on the optimal route selection. In this a mobile node discovers the gateway and updates its route after selecting the optimal path. For this purpose, we propose the following information for gateway discovery. For discovering the gateway, a node relies on gateway advertisement message (GW_ADV). If not, it receives an advertisement message; it has to send a gateway solicitation message (GW_SOL) to Internet gateway via multi-hop neighbours.

\section{Algorithm for Gateway Discovery using PSO}

1. Gateway broadcasts GW_ADV message to the entire mobile node in its proactive zone.

2. if mobile nodes receive a single GW_ADV

Updates its routing table of mobile node else

Performs PSO for getting the optimal route.

\section{end if}

3. if mobile node has a default gateway

if no another GW_ADV received Continue operation

else

Compare the paths based on PSO and update routing table accordingly. end if

4. if nodes do not receive a GW_ADV

Mobile nodes send a multi-hop

GW_SOL to gateway.

end if

5. if gateway reply with a single GW_ADV to source node

Update route according to this message. else

Compare optimal route using PSO and update route accordingly.

6. Repeat Step 1 


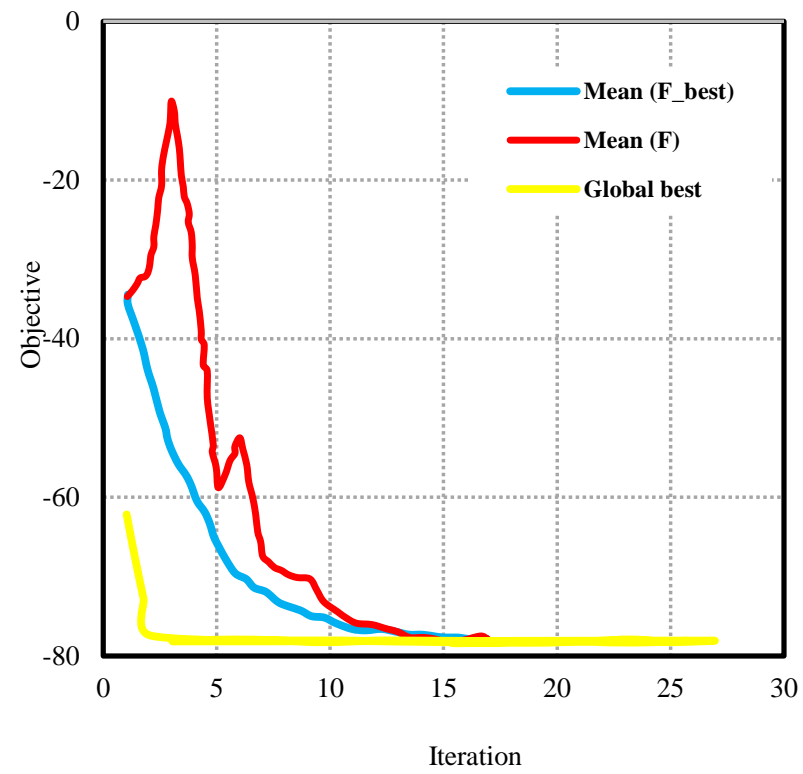

Figure.3 Comparison Graph between Local Best and Global Best Value after Executing Proposed Algorithm

\section{Simulation and result analysis}

We used MATLAB tool to implement Swarm optimization algorithm. First, we execute the Particle Swarm Optimization using proposed fitness function and shown how the particle gets the best value for local best and global best position. Then we have shown two scenarios, where we have first implemented AODV routing protocol to route the packet by shortest path. Then we have compared it to our proposed path selection protocol.

We have shown the graph between our proposed objective and number of iteration in Fig. 3. It shows the particle best position and global best position attained in different number of iterations. The PSO has optimal result, which consist of set nodes between source and destination node to route data packet, when it terminates.

We have considered two scenarios to illustrate the working of our proposed work, which are discussed below. In first scenario, we took 30 number of nodes to find shortest path between source and destination node. Parameters used in first scenario are given in Table 1.

Fig. 4 shows the distribution of nodes in ad-hoc network. As we can discern the nodes are numbered from 1 to 30 . Blue dots are displaying source node and the destination node is red. All supplementary nodes are the intermediate nodes. On this web, we have early requested the shortest trail algorithm utilized by our proposed work.
Table 1. Simulation parameters in scenario 1

\begin{tabular}{|l|l|}
\hline \multicolumn{1}{|c|}{ Parameter } & \multicolumn{1}{c|}{ Value } \\
\hline Number of Nodes & 30 \\
\hline Topography Dimension & $11 \mathrm{mx} 11 \mathrm{~m}$ \\
\hline Traffic Type & CBR \\
\hline Topology & Random \\
\hline Initial Node & 1 \\
\hline Destination Node & 30 \\
\hline Distance & 2.2678 (meters) \\
\hline Energy Consumed & $1.8020 \mathrm{e}+003$ (joules) \\
\hline Network Delay & $596.9317 \mathrm{~ms}$ \\
\hline Elapsed time & $0.000846 \mathrm{~s}$ \\
\hline Path followed & {$[12430]$} \\
\hline
\end{tabular}

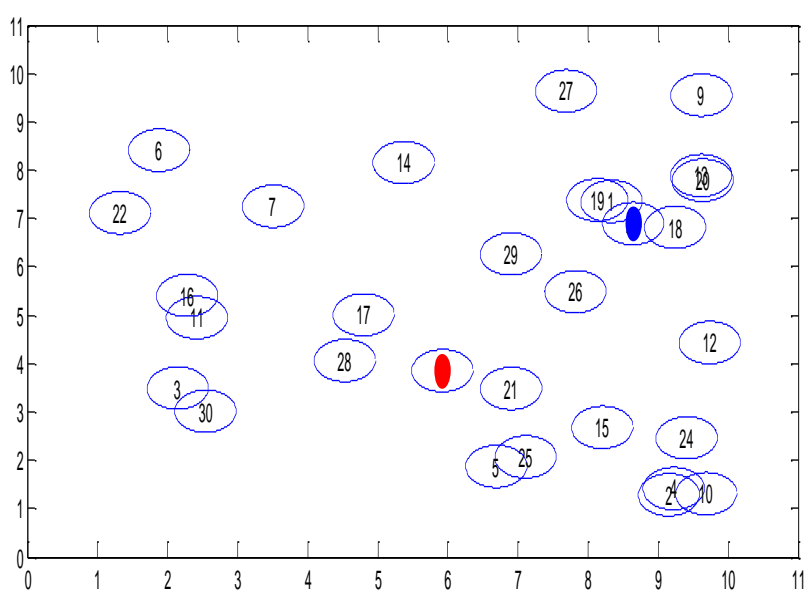

Figure.4 Random number of nodes generated in an AdHoc area

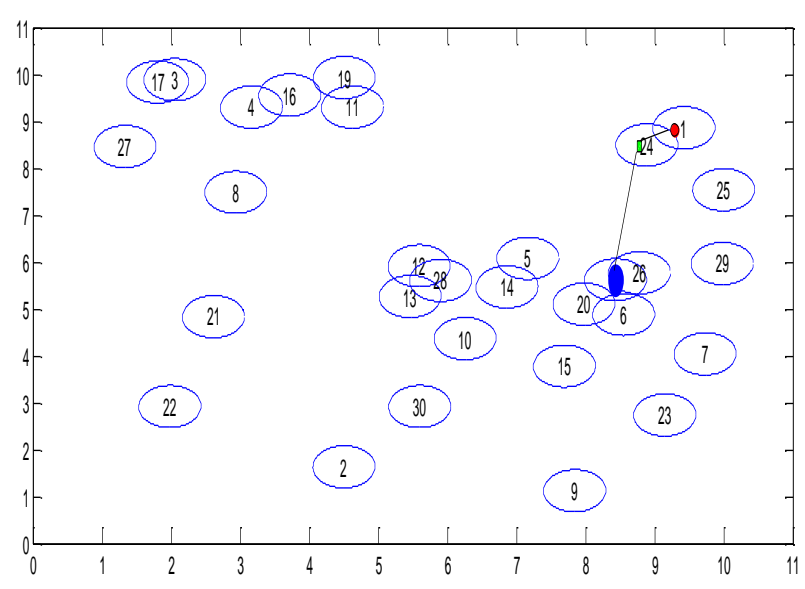

Figure.5 Generated path with 30 nodes using proposed scheme 
We applied our proposed algorithm to find out shortest and load balanced path between source node 1 and destination node 30. Fig. 5 shows that generated path with 30 Nodes with existing scheme. Fig. 5 shows that generated path with 30 nodes using our proposed work, which shows the shortest path is $1=>24=>30$. We took 40 numbers of nodes for second scenario The Parameters used in Second scenario are given in Table 2.

Fig. 6 shows the distribution of nodes in ad-hoc network. As we can discern the nodes are numbered from 1 to 40. Blue dots are displaying source node and the destination node is red. All supplementary nodes are the intermediate nodes.

Table 2. Simulation parameters in scenario 2

\begin{tabular}{|l|l|}
\hline Parameter & Value \\
\hline Number of Nodes & 40 \\
\hline Topography Dimension & $11 \mathrm{mx} 11 \mathrm{~m}$ \\
\hline Traffic Type & CBR \\
\hline Topology & Random \\
\hline Initial Node & 1 \\
\hline Destination Node & 30 \\
\hline Distance & 3.2354 (meters) \\
\hline Energy Consumed & $1.4478 \mathrm{e}+003(\mathrm{joules})$ \\
\hline Network Delay & $1.6904 \mathrm{e}+003 \mathrm{~ms}$ \\
\hline Elapsed time & $0.000892 \mathrm{~s}$ \\
\hline Path followed & {$[1-37-38-11-30]$} \\
\hline
\end{tabular}

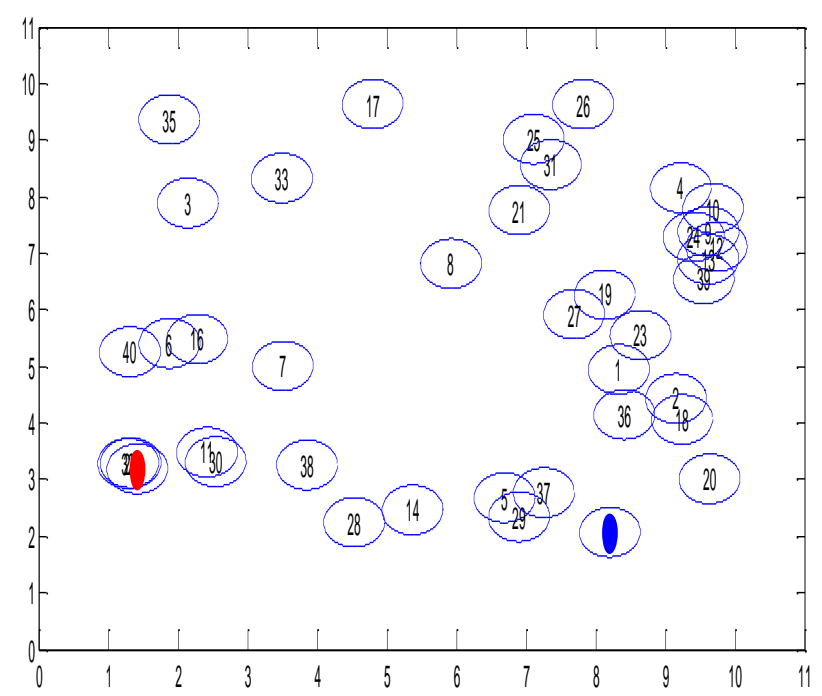

Figure.6 Random number of node generated in Ad-Hoc area

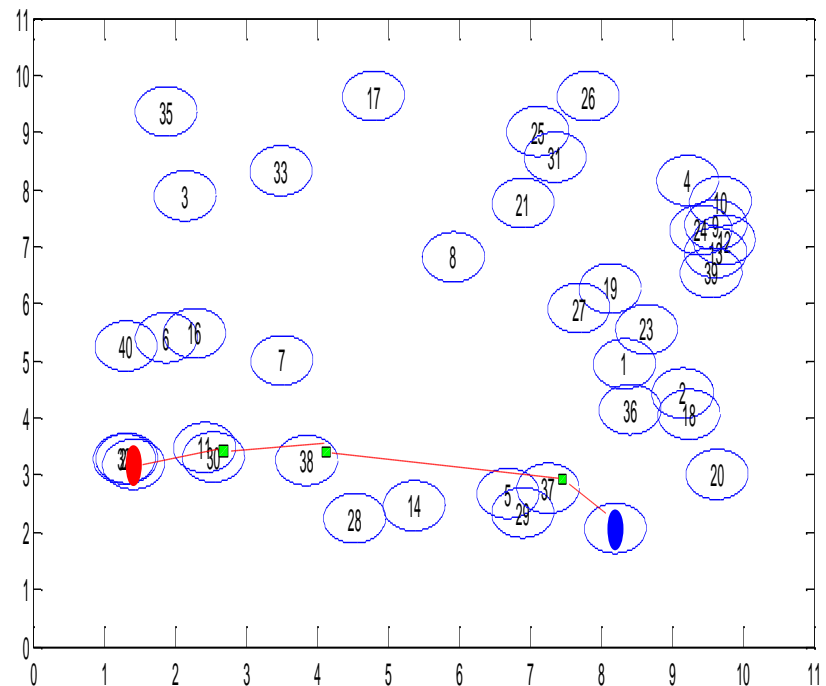

Figure.7 Generated path after executing proposed approach

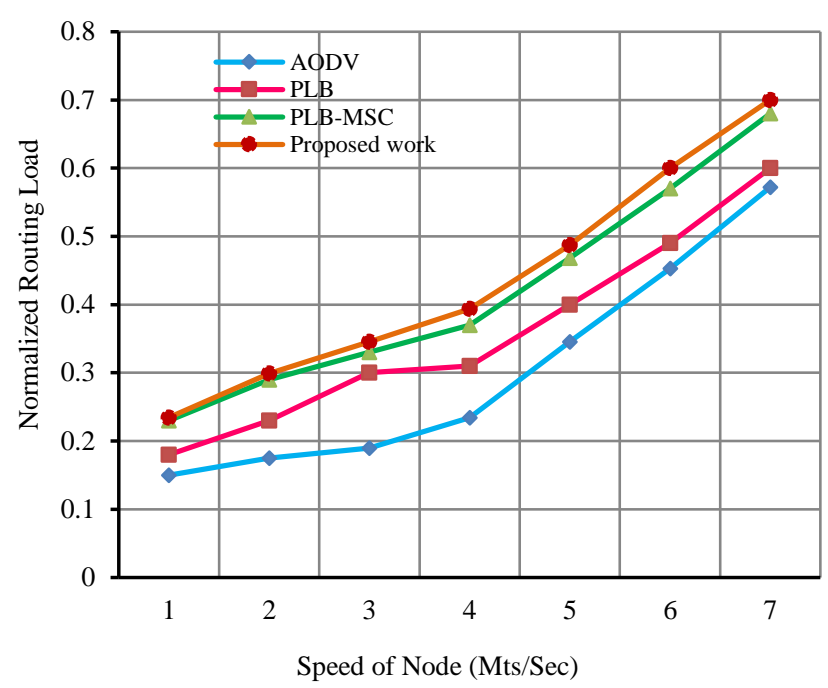

Figure.8 Normalized routing overhead v/s speed of nodes

We can see in Fig.7 how the path is generated when we took 40 numbers of nodes and run the algorithm. The shortest path generated is $1=>37=>38 \Rightarrow 11 \Rightarrow 30$. We have compared our proposed techniques with other approaches like PLB-MSC [14], PLB [15] and AODV [16] protocols. The result is shown in Fig. 8, which shown the simulation result of our proposed work and PLB, PLB-MSC and AODV protocols. Here it is clearly shown that our proposed graph will give better result by selecting more optimal path to route the data packet. Fig.8 shows the comparison of normalized routing overhead with existing approaches.

Graph shown in Fig. 9 is drawn which compares the energy graph of our proposed work and PLBMSC [14], PLB [15] and AODV [16] protocols. Initially, the energy of each node in all the cases was same but as time passes, energy gets depleted. 


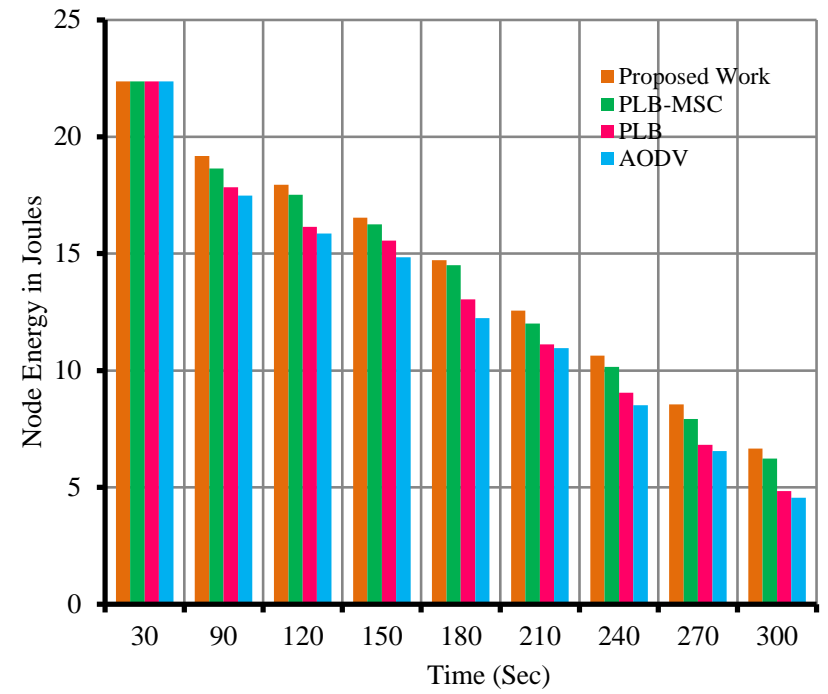

Figure.9 Energy graph

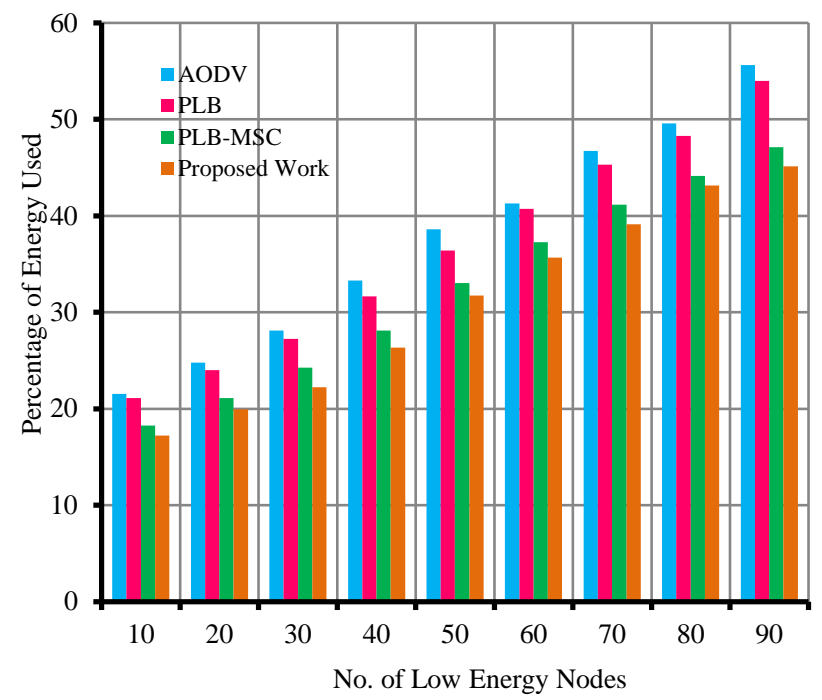

Figure.10 Lifetime

But one can easily interpret that the consumption is fast in case of AODV, PLB and PLB-MSC based approaches in comparison with our proposed one. We have considered a simulation scenario which is run till $300 \mathrm{sec}$ and initial energy in all the cases are 20 joules.

Another comparison is made and represented using graph shown in Fig. 10 for comparing the lifetime of nodes with our proposed work and other existing works like PLB-MSC[14], PLB[15] and AODV[16]. Comparison is done in a varying environment in which the number of low energy nodes is varying in different situations. Outcome reflects that our proposed approach achieves more lifetime than existing approaches.

\section{Conclusion and future scope}

In this paper, we gave our main concern to decrease the overload and remove or solve the problem of congestion which occurs due to path load in ad-hoc networks. So, we surveyed in depth about the many existing packet routing algorithms which select the shortest paths to transfer the data reliably. As ad-hoc networks are dynamic than wired networks where nodes are mobile, thus our challenge was not only to balance the load on the network but also to provide reliable path to the entire mobile nodes. In our proposed work, we have combined PSO with routing algorithm to find the optimized route between source and destination node if the previous selected path gets overloaded or the link is broken, by default it will always select the minimum distance path. Our proposed techniques have been compared with other approaches like PLB-MSC, PLB and AODV protocols. Our approach shows enhancement over existing ones by selecting more optimal path to route the data packet. It is also observed through simulation that our proposed approach achieves more lifetime than existing approaches due to appropriate load balancing technique used as Particle Swarm Optimization is used for selecting an optimal path among all paths available.

In PSO the whole working depends on how we are taking the objective function or fitness function. If one wants more optimized value, then one has to select the more precise function to evaluate the fitness value. To select the shortest path, there are many algorithms which can be applied for mobile ad-hoc network, some of them are already implemented and some are still not considered.

\section{References}

[1] G. Aggelou, "Mobile Ad Hoc Networks: From Wireless LANs to 4G Networks", McGraw-Hill Professional, 2004.

[2] R. Eberhart and J. Kennedy, "A new optimizer using particle swarm theory". In Micro Machine and Human Science MHS'95, In: Proc. of the Sixth International Symposium, pp. 39-43, 1995.

[3] J.H. Zhao, X.Z. Yang, and H.W. Liu, "Loadbalancing Strategy of Multi-gateway for Ad hoc Internet Connectivity", In: Proc. of the International Conference on Information Technology: Coding and Computing (ITCC'05) Vol. 2, pp.592-596, 2005.

[4] A. Trivino-Cabrera, E. Casilari, D. Bartolome, and A. Ariza, "Traffic Load Distribution in Ad Hoc Networks through Mobile Internet Gateways", In: Proc. of Fourth International Working Conference on Performance Modelling 
and Evaluation of Heterogeneous Networks, 2006.

[5] Y.Y. Hsu, Y.C. Tseng, C.C. Tseng, C.F. Huang, J.H. Fan, and H.L. Wu, "Design and Implementation of Two-Tier Mobile Ad Hoc Networks with Seamless Roaming and LoadBalancing Routing Capability", In: Proc. of the First International Conference on Quality of Service in Heterogeneous Wired/Wireless Networks, pp.52-58, 2004.

[6] J. Shin, H. Lee, J. Na, A. Park, and S. Kim, "Load Balancing among Internet Gateways in Ad Hoc Networks", In: Proc. of 62nd IEEE Vehicular Technology Conference, pp.16771680, 2005.

[7] Q. Le-Trung, P.E. Engelstad, T. Skeie, and A. Taherkordi, "LoadBalance of Intra/InterMANET Traffic over Multiple Internet Gateways", In: Proc. of the 6th International Conference on Advances in Mobile Computing and Multimedia, pp. 50-57, 2008.

[8] M. Ali, B. G. Stewart, A. Shahrabi, and A. Vallavaraj. "Congestion adaptive multipath routing for load balancing in Mobile Ad hoc Networks", In: Proc. of Innovations in Information Technology (IIT), 2012 International Conference on, pp. 305-309. IEEE, 2012.

[9] V. Pham, E. Larsen, P. E. Engelstad, and $\varnothing$. Kure. "Performance analysis of gateway load balancing in ad hoc networks with random topologies." In: Proceedings of the 7th ACM international symposium on Mobility management and wireless access, pp. 66-74. ACM, 2009.

[10] A. Rani and M.Dave, "Performance Evaluation of Modified AODV for Load Balancing", Journal of Computer Science, Vol 3, issue 11, pp 863-868, 2007.

[11] Y.H. Yuan, H.M. Chen, and M. Jia, "Adaptive load balancing scheme in ad hoc networks", Journal of Shanghai University (English Edition), Vol. 11, No.3, pp.296-299, 2007.

[12] A.K Gupta, A.K Verma, and H.Sadawarti, "Analysis of various Swarm-Based \& Antbased Algorithm", In: Proc. of ACAI'11, pp. 39-43, 2011.
[13] X. Hu, Y.Shi, and R. Eberhart, "Recent Advances in Particle Swarm", Evolutionary Computation, Vol.1, pp. 90-97, 2004.

[14] R.U. Zaman, K.U.R. Khan and A.V. Reddy, "Path load balanced adaptive gateway discovery in integrated internet-MANET", In: Proc. of Communication Systems and Network Technologies (CSNT), $2014 \quad$ Fourth International Conference on, pp. 203-206, 2014.

[15] K.U.R. Khan, M. Ehthesham, M. Kumar, and R.U. Zaman, "An effective gateway discovery mechanism in an Integrated Internet-MANET (IIM)", In: Proc. of Ultra Modern Telecommunications \& Workshops, 2009. ICUMT'09. International Conference on, pp. 17, 2009.

[16] C. Perkins, E. Belding-Royer and S. Das, "Ad hoc On-Demand Distance Vector (AODV) Routing", RFC 3561, DOI 10.17487/RFC3561, July 2003,

$<$ http://www.rfc-editor.org/info/rfc3561>. 